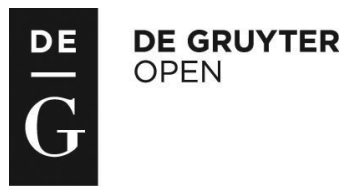

\title{
MYCOLOGICAL PROFILE OF THE INTEGUMENTARY SYSTEM IN FELIN PONIES
}

\author{
Paweł Różański^, Michał Pluta², Dorota Różańska ${ }^{3 \star}$ \\ ${ }^{1}$ Department of Animal and Environmental Hygiene, Faculty of Animal Biology and Breeding, \\ ${ }^{2}$ Department of Horse Breeding and Use, Faculty of Animal Biology and Breeding, \\ ${ }^{3}$ Department and Clinic of Animal Surgery, Faculty of Veterinary Medicine, \\ University of Life Sciences in Lublin, Akademicka 13, 20-950 Lublin, Poland \\ •Corresponding author: d.p.roza@wp.pl
}

\begin{abstract}
Yeast-like microorganisms are frequently isolated from horses. There is a serious interpretation problem with assessment of the health status in the diagnostics of infectious diseases, which is associated with the insufficient knowledge of the composition of the natural flora in the horses' integumentary system. The aim of the investigations was to analyse the yeast-like flora isolated from healthy Felin ponies. The study involved 44 horses aged from 2 to 12 years. The analysed group comprised 28 mares and 16 stallions. The animals were kept in an outdoor rearing system with a possibility of periodic use of a wooden/brick shed. The investigations were conducted in an annual cycle divided into 4 periods corresponding to the seasons of the year. Smears were sampled from horses' nostrils, mouth, ear, back, groin, vagina or foreskin, and collateral groove. Microbiological analysis of the yeast-like strains was performed after plating 1015 samples of material taken from healthy horses. The identification of fungal species was based on their culture characteristics and biochemical profile. Fourteen yeast species were isolated and identified from the sampled material. Candida guilliermondii and Candida albicans 1 were the most prevalent fungi. The study has shown seasonal variability of the composition of the integumentary system microflora in Felin ponies. The greatest number of isolates was obtained from the material sampled from the mouth.
\end{abstract}

Key words: horses, welfare, yeast-like fungi, yeasts, Felin ponies

In addition to pathogenic microorganisms and opportunistic pathogens, many other species of microorganisms accompany humans and animals but do not cause any pathological changes. They can play a positive role in the organism as part of the mucous membrane microbiota of e.g. the gastrointestinal tract. Yeasts constitute an element of intestinal flora. Some of them, e.g. probiotic yeasts, have an ability to inhibit the growth of pathogenic yeasts (Manson et al., 2008; Wang et al., 2014; Weiler and Schmitt, 2003). In cooperation with bacteria, they take part in glucose metabolism in the organisms and exert an impact on the plasma glucose level (Maioli et al., 2008). 
Candida yeasts are a microbial component of the oral microfilm. They can exert an antagonistic effect on Pseudomonas aeruginosa and thus reduce the risk of the growth of this bacterial species. This is particularly interesting, given the fact that opportunistic yeasts are often regarded as a cause of many human and animal diseases (Kraneveld et al., 2012; Shirtliff et al., 2009; Sztajer et al., 2014). As reported by some authors, the mechanism of yeast pathogenicity depends not only on organism immunity and yeast virulence but also on mechanisms of organism's response to infection (Velegraki et al., 2015).

The environment is a natural reservoir of fungi. Pathogenic conditions in immunocompromised organisms can involve multiple organs and systems. The disease develops in animals and humans as a result of uncontrolled proliferation of yeasts after overcoming the defence mechanisms of the organism. The predisposing factors include e.g. immunosuppression, antibiotic therapy, and increased exposure of the organism to environmental fungi (Biegańska, 2006; Dworecka-Kaszak and Adamski, 2005; Mackenthun et al., 2013; Pressler et al., 2003; Różański et al., 2013 a). An important role in the spread of fungal diseases is played by excessive humidity in stables and nutritional deficiencies, in particular in the level of carotene, protein, such trace elements as zinc, iron, and copper, and vitamins A and B (Biegańska, 2008; Cordeiro Rde et al., 2013, 2015; Dworecka-Kaszak, 2008; Stefanetti et al., 2014).

Besides the environmental impact, animals can be a source of infection for humans, and humans can infect animals. Fungi can be transmitted directly by contact or indirectly via tools used by handlers as well as riding equipment (DworeckaKaszak, 2008; Różański et al., 2013 a, b). There are still only preliminary investigations available on the risk of human infections with animal-borne yeasts. However, a possibility of human infection with animal-borne Candida albicans yeasts has been shown. Fungi isolated from humans and animals are quite similar (Edelmann et al., 2005; Hörmansdorfer and Bauer, 2000; Velegraki et al., 2015).

Infection usually develops through the respiratory and digestive systems. In the case of reproductive system infections, an important role in the spread of infection is played by the mode and hygiene of mating the horses. Owing to their great adaptability, fungi readily colonise the host organism and multiply therein. Next, they rapidly spread in the organism via blood and lymph vessels and reach internal organs. The diagnosis of systemic fungal diseases is difficult, as there are no typical clinical symptoms, and delayed diagnosis or misdiagnosis of the disease may lead to animal death. Yeasts have been isolated from human and animal mucous membranes of the mouth, nose/nostrils, conjunctival sac, throat, digestive tract, urinary tract, reproductive tract, and skin. Typically, but not always, the presence of yeast-like microorganisms is associated with diseases (Biegańska, 2008; de Bruijn and Wijnberg, 2004; Dworecka-Kaszak, 2008; Gliński et al., 2011; Ledbetter et al., 2007; Nowak, 2006; Różański, 2013 b; Sgorbini et al., 2008).

The aim of the present study was to perform qualitative analysis of yeast-like microflora of the integumentary system in healthy Felin ponies. 


\section{Material and methods}

The analyses were carried out on material sampled from 44 healthy Felin ponies aged from 2 to 12 years. The group of horses comprised 28 mares and 16 stallions; the animals were kept in an outdoor rearing system with a possibility to use a wood-

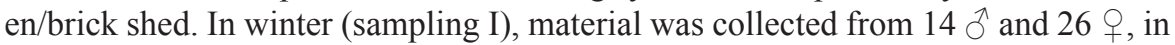
spring (II) from $15 \hat{\sigma}$ and $20 \%$, in summer (III) from $14 \hat{\jmath}$ and $21 \%$, and in autumn (IV) from 15 and 20 . In total, 1015 material samples were analysed throughout the study period.

Horses' diet included hay, oats, and root crops as well as mineral licks in the November-March period. In April-October, the horses grazed on pastures.

During the 2-week period prior to sampling the material for the analyses, the horses did not show any clinical signs of disease and were not subjected to any curative treatment.

The samples were collected four times, i.e. in winter (January), spring (April), summer (June), and autumn (November). The air temperature and humidity on the sampling day were respectively $-6^{\circ} \mathrm{C}$ and $56 \%$ in winter, $3^{\circ} \mathrm{C}$ and $63 \%$ in spring, $19^{\circ} \mathrm{C}$ and $52 \%$ in summer, and $12^{\circ} \mathrm{C}$ and $63 \%$ in autumn.

Smear samples were taken from horses' nostrils, mouth, ear, back, groin, vagina or foreskin, and collateral groove for the analyses. The material obtained after plating the 1015 samples collected from the horses was assessed microbiologically.

The material was stored in sterile tubes on AMIES transport medium and immediately transported to the laboratory. All samples were seeded onto Sabouraud medium and macroscopically homogeneous colonies were obtained by selective screening. The incubation was carried out for 72 hours at a temperature of $30^{\circ} \mathrm{C}$. After incubation, the material was assessed using biochemical assays API 20 C AUX from bioMérieux Polska.

\section{Results}

During the study period, 14 yeast species were isolated from the material, i.e. C. guilliermondii (24.44\%), C. albicans 1 (20\%), C. krusei (8.89\%), C. inconspicua (8.89\%), C. norvegensis (2.22\%), C. lusitanae (4.44\%), C. sp. (6.67\%), Trichosporon asahii (8.89\%), Trichosporon mucoides (2.22\%), Rhodotorula minuta (4.44\%), Rhodotorula sp. (2.22\%), Stephanoascus ciferrii (2.22\%), Geotrichum klebahnii $(2.22 \%)$, and Kodamaea ohmeri $(2.22 \%)$ - Figure 1.

In total, 1015 samples were collected, i.e. 280 in the winter period and 245 in each of the spring, summer, and autumn periods.

Only 2 isolates of $C$. guilliermondii yeasts were detected in the 280 samples collected in winter. In the samples collected in spring, 4 yeast species were isolated, i.e. C. guilliermondii, C. norvegensis, C. lusitanae, and Stephanoascus ciferrii. C. albicans 1 (38.10\%), C. guilliermondii and Trichosporon asahii (19.05\% each), Rhodotorula minuta (9.52\%), as well as C. lusitanaea, Trichosporon mucoides, and 
Rhodotorula sp. (4.76\% each) were the most prevalent yeast species isolated in summer. The most frequent species among the isolates detected in autumn included C. guilliermondii, C. krusei, and C. inconspicua (22.22\% each). Candida sp. accounted for $16.67 \%$ of all the isolates. The isolated C. albicans 1, Kodamaea ohmeri, and Geotrichum klebahnii represented 5.56\% each (Tables 1 and 2).
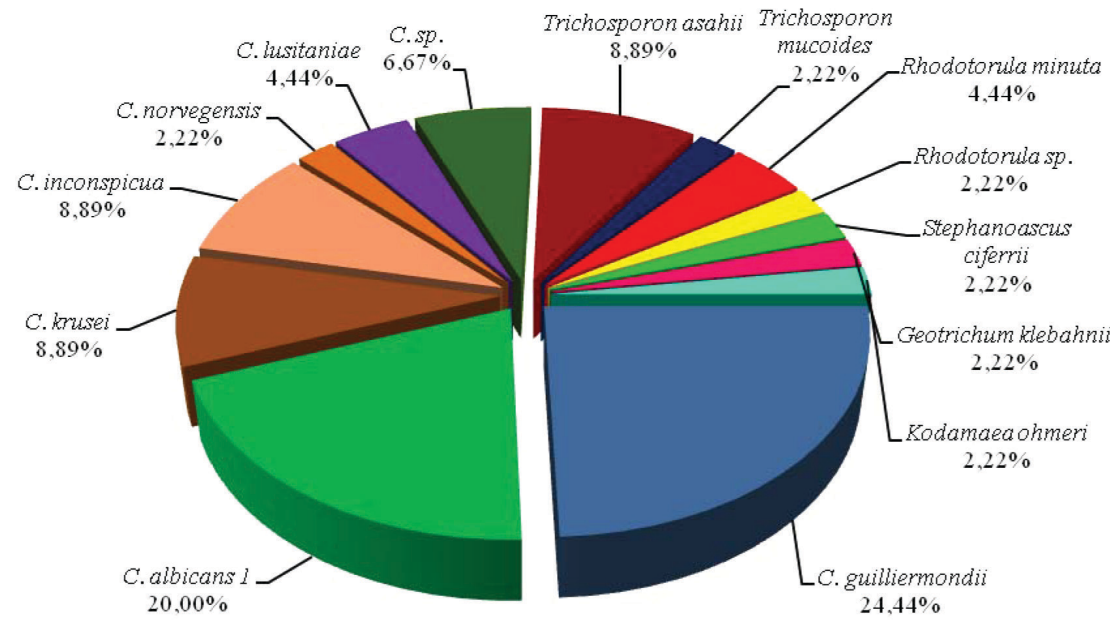

Figure 1. Frequency of occurrence of the fungi throughout the year (in all horses)

In total, $54 \%$ of the yeasts were isolated from the mares. Ten yeast species, with predominance of C. guilliermondii (27.27\%) and C. albicans 1 (22.73\%), were isolated from the 87 mares examined during the entire year.

The greatest number of isolates from the mares was obtained from the material sampled in summer. Yeast species that were isolated during the individual seasons and throughout the year are presented in detail in Table 1. Eight yeast species were isolated during the study year from the 58 examined stallions. C. guilliermondii (22.73\%), followed by C. albicans 1, C. krusei, and C. inconspicua (18.18\% each) were isolated most frequently. C. sp. accounted for $9.09 \%$, and C. lusitaniae, Geotrichum klebahnii, and Stephanoascus ciferrii represented $4.55 \%$ each.

The greatest number of isolates from the stallions was obtained from the material sampled in autumn (14) and summer (6). One isolate was detected in each of the winter and spring periods (Table 2). The strains differed in terms of the quantity and quality between the horse males and females (Figure 2). Similar differences were also noted in relation to the different material sampling sites (Figure 3) and the seasons of the year (Figure 4). 
Table 1. Prevalence of yeast-like microorganisms in the individual samples from the mares

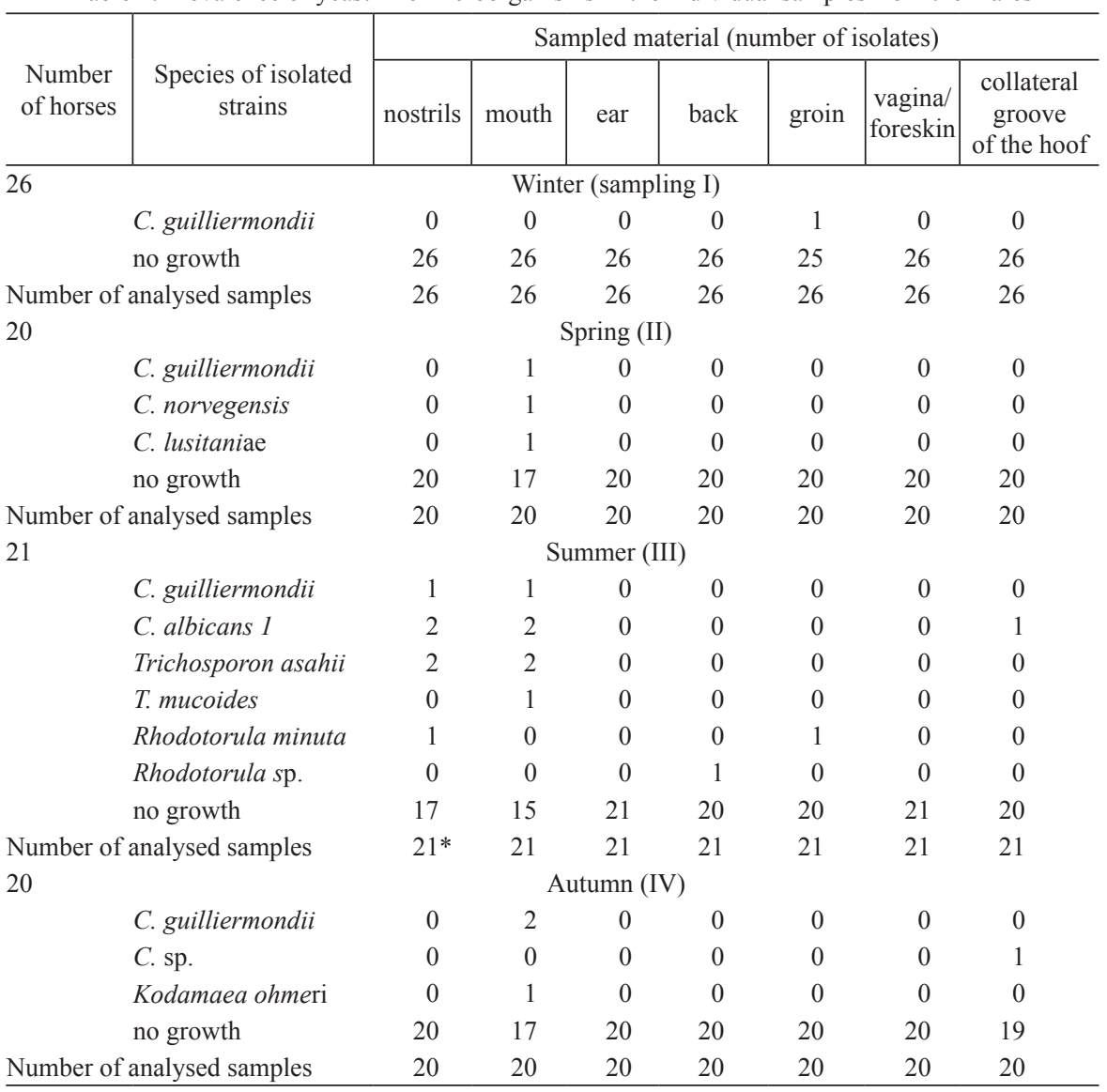

* more than 1 strain was obtained from some isolates.

Table 2. Prevalence of yeast-like microorganisms in the individual samples from the stallions

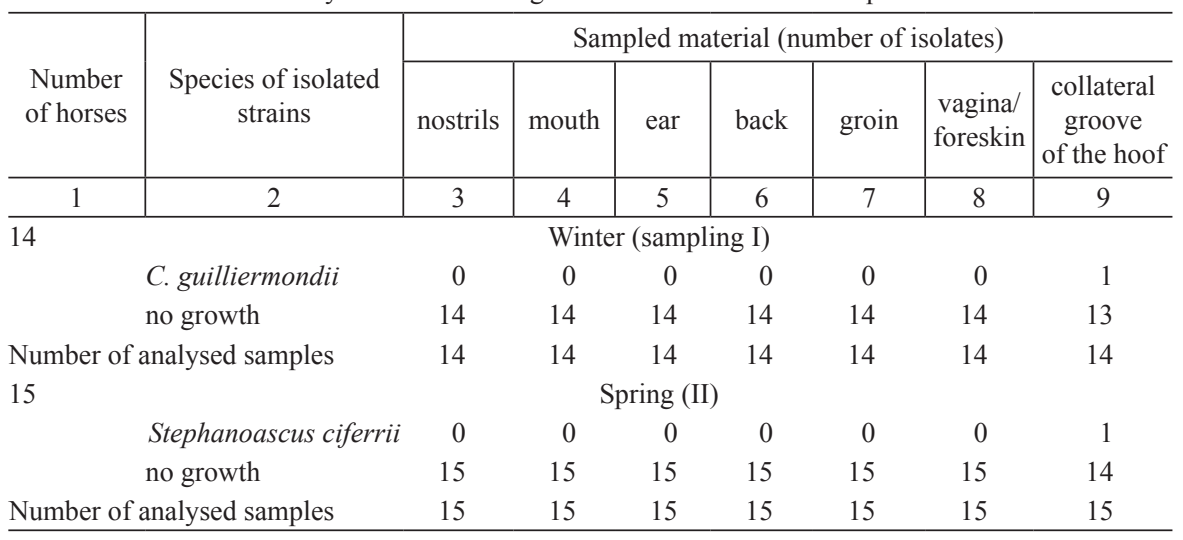


Table 2 - contd.

\begin{tabular}{|c|c|c|c|c|c|c|c|c|}
\hline 1 & 2 & 3 & 4 & 5 & 6 & 7 & 8 & 9 \\
\hline \multicolumn{9}{|c|}{ Summer (III) } \\
\hline & C. guilliermondii & 0 & 1 & 0 & 0 & 1 & 0 & 0 \\
\hline & C. albicans 1 & 0 & 2 & 0 & 1 & 0 & 0 & 0 \\
\hline & C. lusitaniae & 0 & 0 & 0 & 0 & 0 & 1 & 0 \\
\hline & no growth & 14 & 11 & 14 & 13 & 13 & 13 & 14 \\
\hline \multicolumn{2}{|c|}{ Number of analysed samples } & 14 & 14 & 14 & 14 & 14 & 14 & 14 \\
\hline \multirow[t]{8}{*}{15} & \multicolumn{8}{|c|}{ Autumn (IV) } \\
\hline & C. guilliermondii & 1 & 0 & 0 & 0 & 0 & 0 & 1 \\
\hline & C. albicans 1 & 0 & 1 & 0 & 0 & 0 & 0 & 0 \\
\hline & C. krusei & 0 & 0 & 1 & 0 & 0 & 0 & 3 \\
\hline & C. inconspicua & 0 & 0 & 1 & 0 & 0 & 0 & 3 \\
\hline & C. sp. & 1 & 1 & 0 & 0 & 0 & 0 & 0 \\
\hline & Geotrichum klebahnii & 0 & 1 & 0 & 0 & 0 & 0 & 0 \\
\hline & no growth & 13 & 12 & 14 & 15 & 15 & 15 & 11 \\
\hline \multicolumn{2}{|c|}{ Number of analysed samples* } & 15 & 15 & $15^{*}$ & 15 & 15 & 15 & $15^{*}$ \\
\hline
\end{tabular}

* more than 1 strain was obtained from some isolates.

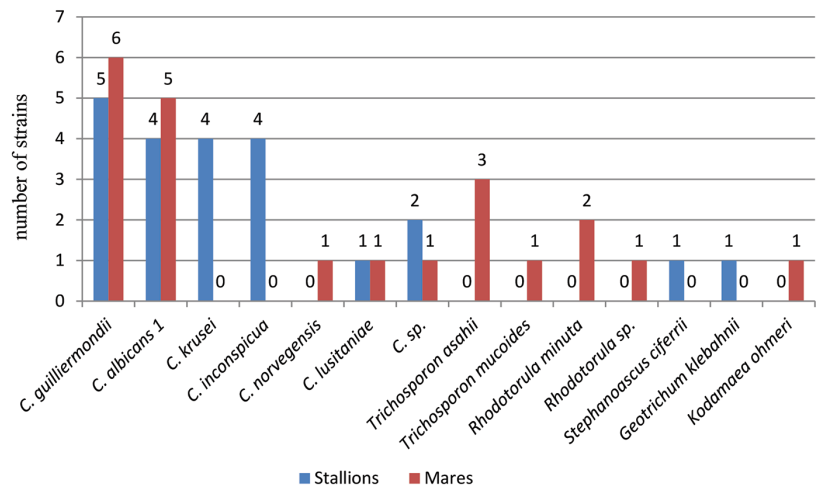

Figure 2. Comparison of isolates of the individual strains throughout the study period

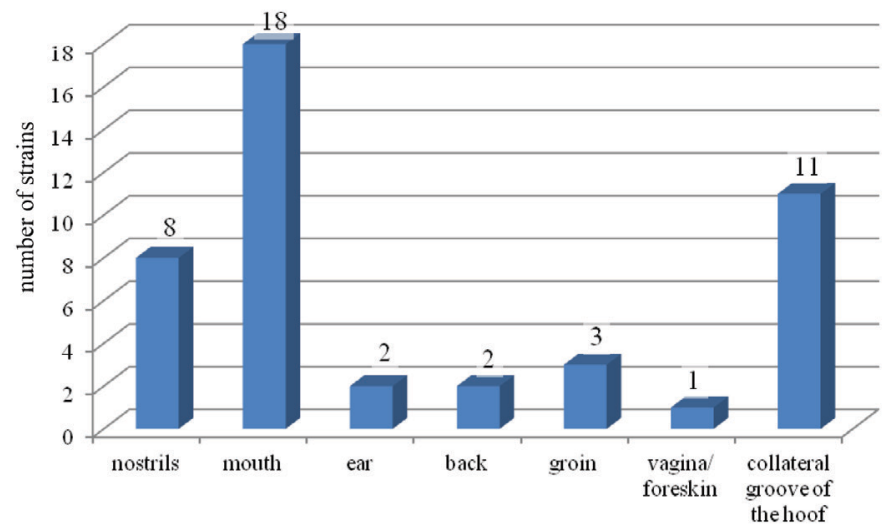

Figure 3. Comparison of isolated fungal species from the individual material sampling sites 


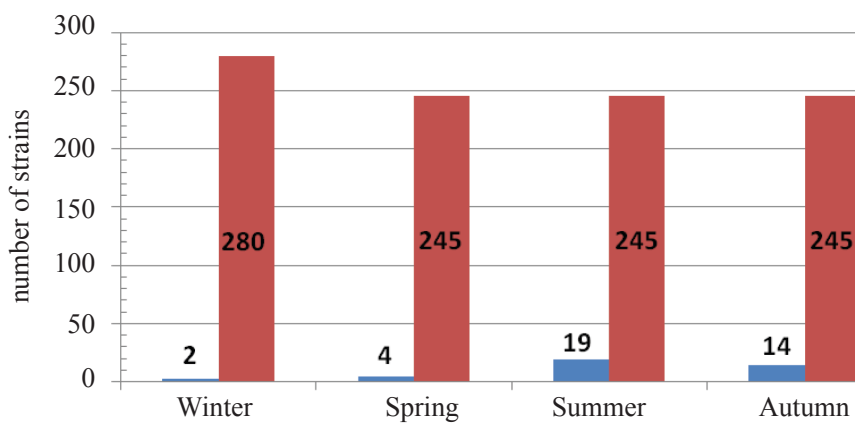

Figure 4. Comparison of the number of isolated fungi and the number of analysed samples in the individual seasons of the year

\section{Discussion}

Fungi are a natural component of the microflora of living organisms. The isolation of the material presented in this article confirms reports provided by other authors (Arendrup, 2013; Pacyńska, 2013; Różański et al., 2013 b).

However, it is difficult to refer directly to the percentage of the individual strains in the total pool of isolates reported by other researchers, due to the differences in the study objectives and, accordingly, differences in the research models employed (Khosravi et al., 2014; Reilly and Palmer, 1994; Cordeiro et al., 2013; Brilhante et al., 2016).

It should be emphasised that the results obtained in the present study refer to analysis of material sampled from healthy horses of the same breed kept in identical environmental conditions. In the scanty literature in this field, there are only few reports of analysis of material originating from a homogeneous breed group (Różański et al., 2013 b). In addition, this is the first report of the fungal flora in the Felin pony breed. The available literature does not provide reports of fungal flora in horses kept in an outdoor rearing system during the entire year. The microflora of the human oral cavity can comprise many different fungal species. Yeast from the genera Candida, Cladosporium, Aureobasidium, Saccharomyces, and Cryptococcus have been isolated most frequently (Ghannoum et al., 2010). The skin fungal microbiota of healthy humans is dominated by Malassezia (Luciana et al., 2006; Gao et al., 2010; Paulino et al., 2006). The diversity of the microbiota of individual parts of the gastrointestinal tract varies significantly and depends on environmental factors, diet and health status. However, it is difficult to determine unambiguously its normal composition (Tappenden and Deutsch, 2007; Mättö et al., 2005). In total, 13 yeast species were isolated in material from 60 nymphs (Nymphicus hollandicus). The most frequently isolated species were represented by $C$. albicans (32.5\% isolates), C. tropicalis (20\%), T. asteroides (12.5\%), and C. famata (10\%) (Brilhante et al., 2010). In cattle, yeasts are mainly isolated from the mammary gland. However, these reports present investigations of infected animals only (Krukowski et al., 2000, 2006). 
In terms of the relationship between the seasons of the year and the number of isolates obtained, the results confirm the observations reported by other authors (Pacyńska, 2013).

The increased number of the yeasts on horses' skin and mucous membranes should be regarded as typical for the warmer periods of the year. In the present study, C. guilliermondii and C. albicans 1 were isolated most frequently. This confirms the substantial proportion of the Candida genus strains described in the literature (Arendrup, 2013; Pacyńska, 2013; Różański et al., 2013 b).

In the analysed research results, attention should be paid to the relatively small number of the isolated strains, which is noticeably different from results reported in other papers. No publications show results of examination of horses kept in conditions that would be so similar to natural ones. Importantly, Felin ponies originate from horses that are close to ancient breeds, which is confirmed by the good health condition of the representatives of this group. The small number of the isolated strains may indicate an important role of the breeding environment conditions in the composition of the animal organism microbiota (Arendrup, 2013; Różański et al., 2013 b). This thesis is supported by the fact that the greatest number of isolates was obtained from the material sampled from the mouth and the collateral groove, i.e. sites in contact with the environment, and the lowest number was isolated from vaginal and/or foreskin samples, in which the microflora is largely determined by the defence mechanisms of the organism.

Biochemical fungal identification is a well proven and widely used method, however, given the development of analytical techniques, identification can be based on molecular methods, which are undoubtedly conclusive. This is particularly advisable when results indicate low or uncertain level of differentiation.

\section{Conclusions}

Yeasts are part of the natural microbiota of horses' organisms. Their presence does not always imply an ongoing disease. The present results indicate an important role of the breeding environment in determination of the composition of horses' microbiota. It should also be emphasised that, due to the lack of analogous research reports in the literature, the experimental model described in this paper can constitute a reference for future identifications.

The need for maintenance of horses in welfare-friendly conditions focused on determination of optimal breeding environment parameters is underlined in this study. It is also a rationale for the developed research model.

\section{References}

Arendrup M.C. (2013). Candida and candidaemia. Susceptibility and epidemiology. Dan. Med. J., 60: B4698, 1-32.

B i e g a ń s k a M.J. (2006). Candida yeasts - not always pathogenic but .... (in Polish). Mikologia Lekarska, 13: 318-321.

B i e g án s k a M.J. (2008). Infections with Candida genus fungi in animals (in Polish). Mikologia Lekarska, 15: 155-159. 
Brilhante R.S., Bittencourt P.V., Castelo-Branco Dde S., de Oliveira J.S., Alenc ar L.P., Cordeiro Rde A., Pinheiro M., Nogueira-Filho E.F., Pereira-Neto W d e A., S i d r i m J.J., R o c h a M.F. (2016). Trends in antifungal susceptibility and virulence of Candida spp. from the nasolacrimal duct of horses. Med. Mycol., 54: 147-154.

B rilhante R.S.N., C a s t e $10-$ B ranco D.S.C.M., S o a res G.D.P., A s te t e-M e d r a no D.J., Monteiro A.J., Cordeiro R.A., S i drim J.J.C., R o cha M.F.G. (2010). Characterization of the gastrointestinal yeast microbiota of cockatiels (Nymphicus hollandicus): a potential hazard to human health. JMM, 59: 718-723.

Cordeiro Rde A., Bittencourt P.V., Brilhante R.S., Teixeira C.E., Castelo-Branco Dde S., Silva S.T., De Alencar L.P., Souza E.R., Bandeira Tde J., Monteiro A.J., S i dri m J.J., R o c h a M.F. (2013). Species of Candida as a component of the nasal microbiota of healthy horses. Med Mycol., 51: 731-736.

Cordeiro Rde A., de Oliveira J.S., Castelo-Branco Dde S., Teixeira C.E., Marques F.J., Bittencourt P.V., Carvalho V.L., Bandeira Tde J., Brilhante R.S., Moreira J.L., P ereira-Net o W de A., S idrim J.J., R o c ha M.F. (2015). Candida tropicalis isolates obtained from veterinary sources show resistance to azoles and produce virulence factors. Med. Mycol., 1: 145-152.

d e B ruij n C.M., W i j n b e rg I.D. (2004). Potential role of Candida species in antibiotic-associated diarrhoea in a foal. Vet. Rec., 3: 26-28.

D w o r c ka-Kas zak B. (2008). Animals as a potential source of fungal infections in humans (in Polish). Wiad. Parazytol., 54: 101-108.

D w o r e c k a - K a s z a k B., A d a m s k i Z. (2005). Infections with Malassezia genus fungi (in Polish). SGGW, Warszawa.

E d e $1 \mathrm{~m}$ a n n A., Kruger M., S ch m id J. (2005). Genetic relationship between human and animal isolates of Candida albicans. J. Clin. Microbiol., 43: 6164-6166.

G a o Z., P e re z - P e re z G.I., Chen Y., B l a s e r M.J. (2010). Quantitation of major human cutaneous bacterial and fungal populations. J. Clin. Microbiol., 48: 3575-3581.

Ghannoum M.A., Jurevic R.J., Mukherjee P.K., Cui F., Sikaroodi M. (2010) Characterization of the oral fungal microbiome (mycobiome) in healthy individuals. PLoS Pathog., 6: e1000713, doi:10.1371/journal.ppat.1000713

Gliński Z., Kostro K., Wołoszyn S., Gajęcki M. (2011). Mycoses and mycotoxicoses in animals (in Polish). UP, Lublin.

Hörmans dorfer S., B a u e r J. (2000). Yeast infections in veterinary medicine. Contrib. Microbiol., 5: 54-78.

Khosravi A.R., Nikaein D., Sharifzadeh A., Gharagozlou F. (2014). Ocular fungal flora from healthy horses in Iran. J. Mycol. Med., 24: 29-33.

Kraneveld E.A., Buijs M.J., B onder M.J., Vis s er M., Ke ij s e r B.J.F., Cri elaard W., $\mathrm{Z}$ a u r a E. (2012). The relation between oral Candida load and bacterial microbiome profiles in Dutch older adults. PLoS One., http://dx.doi.org/10.1371/journal.pone.0042770

Krukowski H., Tietze M., Majewski T., Różański P. (2000). Survey of yeast mastitis in dairy herds of small-type farms in the Lublin region, Poland. Mycopathologia, 150: 5-7.

Kruk ow sk i H., L i s ow s k i A., Różań s k i P., S k órk a A. (2006). Yeast and alga isolated from cows with mastitis in the south-eastern part of Poland. Pol. J. Vet. Sci., 9: 181-184.

L e d b e t t e r E.C., P a t t e n V.H., S c a r l e $t \mathrm{t}$ J.M., Ver m e y l e n F.M. (2007). In vitro susceptibility patterns of fungi associated with keratomycosis in horses of the northeastern United States: 68 cases (1987-2006). J. Am. Vet. Med. Assoc., 1: 1086-1091.

Luci a n a C. P a u lin o L.C., Ts en g C., S trober B.E., B l a s e r M.J. (2006). Molecular analysis of fungal microbiota in samples from healthy human skin and psoriatic lesions. J. Clin. Microbiol., 44: 2933-2941.

M a c k e n th u n E., C o e n e n M., Ve r v u e r t I. (2013). Effects of Saccharomyces cerevisiae supplementation on apparent total tract digestibility of nutrients and fermentation profile in healthy horses. J. Anim. Physiol. Anim. Nutr. (Berl)., 97, Suppl. 1: 115-120.

Maioli M., Pes G.M., Sanna M., Cherchi S., Dettori M., Manca E., Farris G.A. (2008). Sourdough-leavened bread improves postprandial glucose and insulin plasma levels in subjects with impaired glucose tolerance. Acta Diabetol., 45: 91-96. 
Man s on J.M., R a u ch M., G i l m or e M.S. (2008). The commensal microbiology of the gastrointestinal tract. Adv. Exp. Med. Biol., 635: 15-28.

Mät tö J., M a unukse la L., Ka jand er K., P a lva A., Korpela R., K a s sinen A., S a are la M. (2005). Composition and temporal stability of gastrointestinal microbiota in irritable bowel syndrome - a longitudinal study in IBS and control subjects. FEMS Immunol. Med. Microbiol., 43: 213-222.

N ow a k G. (2006). Equine mycosis - Current problems (in Polish). Wet. Prakt., 6: 70-76.

P a c y ń s k a J.A. (2013). Yeast-like fungi and yeasts isolated from healthy breeding horses. Pol. J. Vet. Sci., 16: 69-76.

Paulino L.C., Tseng C.H., Strober B.E., B laser M.J. (2006). Molecular analysis of fungal microbiota in samples from healthy human skin and psoriatic lesions. J. Clin. Microbiol., 44: 2933-2941.

Pre s s 1 e r B.M., Va d e n S.L., L a n e I.F., C o w g i 11 L.D., D y e J.A. (2003). Candida spp. urinary tract infections in 13 dogs and seven cats: predisposing factors, treatment and outcome. J. Am. Anim. Hosp. Assoc., 39: 263-270.

Re illy L.K., P a 1 m er J.E. (1994). Systemic candidiasis in four foals. J. Am. Vet. Med. Assoc., 1: 464-466.

R ó ża ń s k i P., Śla s k a B., Ró ża ń s k a D. (2013 a). Current status of prevalence of yeast-like fungi in the environment of horses bred in Poland. Ann. Anim. Sci., 13: 365-374.

Różański P., Śl a ska B., Różańska D. (2013 b). Prevalence of yeasts in English full blood mares. Mycopathologia, 175: 339-344.

Sgorbini M., Barsotti G., Nardoni S., Mancianti F., Rossi S., Corazza M. (2008). Fungal flora of normal eyes in healthy newborn foals living in the same stud farm in Italy. J. Equine Vet. Sci., 28: 540-543.

Sh i r t l i ff M.E., P e t e r s B.M., J a b r a - R i z k M.A. (2009). Cross-kingdom interactions: Candida albicans and bacteria. FEMS Microbiol Lett., 299: 1-8.

Stefanetti V., Marenzoni M.L., Lepri E., Coletti M., Casagrande Proietti P., Agnetti F., Crotti S., Pitzurra L., Del Sero A., Passamonti F. (2014). A case of Candida guilliermondii abortion in an Arab mare. Med. Mycol. Case Rep., 3: 19-22.

Sztajer H., S zafranski S.P., Tomas ch J., Reck M., Nimtz M., Rohde M., Wagner- D ö b l e r I. (2014) Cross-feeding and interkingdom $\underline{\text { communication }} \underline{\text { in }} \underline{\text { dual-species }} \underline{\text { biofilms }}$ of Streptococcus mutans and Candida albicans. ISME J., 8: 2256-2271.

T a p p e nd e n K.A., D e u t s ch A.S. (2007). The physiological relevance of the intestinal microbiotacontributions to human health. J. Am. Coll. Nutr., 26: 679-683.

Velegraki A., C a farchia C., Gait an is G., I at t a R., B o ek hout T.(2015). Malassezia infections in humans and animals: pathophysiology, detection, and treatment. PLoS Pathog., 11: e1004523.

Wa ng Z.K., Yang Y.S., S te f k a A.T., S u n G., P e n g L.H. (2014). Review article: fungal microbiota and digestive diseases. Aliment. Pharm. Therap., 39: 751-766.

W e i 1 e r F., S c h m it t M.J. (2003). Zygocin, a secreted antifungal toxin of the yeast Zygosaccharomyces bailii, and its effect on sensitive fungal cells. FEMS Yeast Res., 3: 69-76.

Received: 23 VI 2016

Accepted: 17 II 2017 